\title{
Comparison of Aircraft Conceptual Design Weight Estimation Methods to the Flight Optimization System
}

\author{
Bryce L. Horvath ${ }^{1}$ \\ NASA Langley Research Center, Hampton, VA, 23681 \\ and \\ Douglas P. Wells ${ }^{2}$ \\ Formerly of NASA Langley Research Center, Hampton, VA, 23681
}

\begin{abstract}
Weight estimation is critical in the aircraft conceptual design process. The Flight Optimization System (FLOPS) is an aircraft conceptual design tool that has been the primary aircraft synthesis software used by the Systems Analysis and Concepts Directorate at NASA Langley Research Center. FLOPS includes multiple modules that represent aircraft design disciplines. The FLOPS weight module includes estimation methods that are similar in nature to other regression based aircraft preliminary weight estimation methods, however the FLOPS methods were created to use a minimum number of input parameters to limit the effort required by the designer to apply it. As FLOPS has recently been made publically available, this work compares the FLOPS weight estimation methods with several similar methods with the goal of explaining the differences in FLOPS, providing conceptual designers with a brief introduction to the method before attempting to apply it, and providing a reference to inform the development of future weight estimating relationships. In this paper, the Boeing 737-200 is used as a test case to highlight to differences and similarities in the methods.
\end{abstract}

\section{Introduction}

$\mathrm{C}$ ONCEPTUAL aircraft design is the critical phase of the aircraft development process where the first analyses and performance estimates are conducted. Manufacturers use the resulting weight and performance estimates to plan budgets and schedules for production projects. The success of a production project depends heavily on the quality of the conceptual design phase analyses. The more information and data available to the conceptual aircraft designer, the better quality product that can be produced, potentially saving significant schedule and cost throughout the project.

The conceptual design phase often starts with some level of weight estimation. It involves some analytical sizing or estimating the appropriate gross weight and size of the aircraft. Weight estimation is a critical process in conceptual aircraft design because it is the basis for cost estimation and influences the total project cost [1]. Therefore, it is in the best interest of airframe manufacturers and designers to improve the accuracy of conceptual design weight estimation methods and the data available at this phase.

Regression-based or semi-empirical weight estimation is desired at the conceptual level to enable a design space exploration in a short period of time. This enables the designer to eliminate infeasible or expensive designs, in terms of weight, early in the design process. Weight estimating regressions are relationships or equations that estimate weight based on parameters from historical data. This paper examines several popular, publicly available weight estimation methods and compares them to the methods in NASA Flight Optimization System (FLOPS) program [2], explaining the process used by each method to achieve a weight breakdown for conceptual design. In addition, several weight estimating relationships are examined more closely to illustrate the similarities and differences. The objective of making these comparisons is to inform designers of the various features of each methodology, provide a brief

\footnotetext{
${ }^{1}$ Aerospace Engineer, Vehicle Analysis Branch.

${ }^{2}$ Aerospace Engineer, AIAA Senior Member.
} 
introduction to the FLOPS weight estimation method, and to provide a reference to inform the development of future weight estimating relationships.

\section{Weight Estimation Methods}

The weight estimation methods chosen for examination in this study include the methods from FLOPS [2,3], Raymer [4], and Roskam [5]. Unfortunately, most public regression-based weight estimation methods are based on aircraft that were designed in the 1960s to the 1970s, or even earlier. Few have modifications that attempt to capture the advances in materials and design. For this reason, and the availability of public data, the Boeing 737-200, which entered service in 1968, was chosen as the example case for this study. The overall process and level of effort for each method are explained in their respective subsections.

\section{A. FLight OPtimization System (FLOPS)}

FLOPS is an aircraft sizing and synthesis program developed by Linwood (Arnie) McCullers at NASA Langley Research Center in Hampton, VA [3]. Development of the method began in the 1980s and ended in 2011. FLOPS was created to design and synthesize the configuration of new aircraft at the conceptual design level and assess the impacts of advanced technologies. FLOPS is a single computer program with an execution control module that executes eight other modules. These modules include weights analysis, aerodynamics analysis, engine cycle analysis, propulsion data scaling and interpolation, detailed mission performance analysis, takeoff and landing performance analysis, noise footprint analysis, and cost analysis. This paper is focused only on the weight estimation module in FLOPS. More information about FLOPS and its analysis modules can be found in Ref [2].

The overarching philosophy used in the development of the weights module was to use as few input parameters as possible to perform a complete weight buildup. Furthermore, when there is a requirement for a parameter that is difficult to calculate early in the conceptual design phase, provide methods to estimate that parameter. The methodology was calibrated using data from 17 transport aircraft and 25 fighter/attack aircraft. Additionally, the wing weight estimation method was calibrated with additional data generated by the Aeroelastic Tailoring and Structural Optimization (ATSO) program [6] to extend its applicable range beyond the aircraft that existed at the time.

The weight of each aircraft component is estimated using weight estimating relationships based upon the aircraft geometry and an initial estimate of gross weight. The component weights are summed to calculate a zero fuel weight. FLOPS can be executed in two modes. If the gross weight is specified, the mission fuel is calculated and FLOPS performs the mission analysis and estimates the range. If the range is specified instead of the gross weight, FLOPS will perform the mission analysis and iterate until the mission fuel matches the fuel required to meet the minimum range constraint. At the same time, FLOPS sizes the aircraft, which results in corresponding gross weight and component weights required for the design mission.

A selection of the weight estimation methods from FLOPS are described further in this document. However, as a full description of the methods in FLOPS is beyond the scope of this work the reader is encouraged to refer to Ref. [3] for more details. This reference includes a complete description of the FLOPS weight estimation methods for transport, hybrid wing body (HWB), fighter/attack, and general aviation aircraft along with methods for predicting the tail volume coefficients and for sizing commercial transport and HWB fuselages based on the number of passengers in each class.

\section{B. Raymer}

The Raymer method is defined in the book Aircraft Design: A Conceptual Approach [4]. The textbook is intended to guide the designer through the aircraft design process starting from an initial idea. Raymer includes three processes to estimate the aircraft weight in this method. Each method progressively builds up knowledge about the aircraft to provide a better estimate of aircraft weight.

In the first process the aircraft gross weight is estimated using specified crew and payload weight, an empty weight regression curve, and a fuel fraction from a mission analysis based on segment fuel fractions as a ratio to the gross weight. The second process is intended to provide a more accurate estimate than the first. This method estimates the empty weight using basic statistical weight fractions. The structural weights are based on exposed planform areas, gross weight, and engine weight.

The third process is intended to provide a more accurate weight estimate than the second. As it is similar in complexity to the FLOPS method it is the focus of the Raymer portion of this paper. In the third process individual component and subsystem weights are estimated using regression equations. These regression equations are functions of properties of the aircraft. The designer can sum the results of these regression equations to estimate component, subsystem, and overall system weight. Typically, this approach involving the summation of the components generally 
provides a more accurate empty weight estimate than other methods and has the added benefit of allowing the designer to distribute weights on the aircraft to estimate aircraft mass properties.

As will be discussed in the following section, overall the Raymer third process requires more complex input parameters than the others. However, as this weight estimation is part of a larger aircraft design process numerous methods are defined to estimate these value and there are numerous recommendations for how to adjust weights to fit specific concepts.

\section{Roskam}

The Roskam method is defined in the multivolume series Airplane Design [5], which like Raymer is intended to guide the designer through the process of developing a conceptual aircraft design. Three methods for estimating aircraft weight are provided, each building on the previous method to improve the fidelity of the estimate. The first process is used to estimate the gross, fuel, and empty weight. The second process is called the Class I method and utilizes the results from the first process to estimate component weights based on historical data of similar aircraft. The third process is called the Class II method and uses more detailed aircraft data to perform a detailed weight estimate of the aircraft components based on regression equations. As the third process is comparable in complexity to the FLOPS method, it is the focus of the comparison in this paper.

The first process estimates the payload weight and crew weights. For a commercial transport aircraft these are based on the number of passengers and Federal Aviation Regulations (FAR). The gross weight is estimated and can be based on similar aircraft. The fuel weight is estimated through a mission analysis based on segment fuel fractions as a ratio to the gross weight. The empty weight is estimated using a linear relationship to the gross weight.

The second, or Class I, process estimates the component weights. Weight fractions are calculated based on aircraft with similar geometry and mission requirements. The weight fractions are relative to the gross weight. Once the component weights are calculated, they are summed to result in the empty weight.

The third process, or Class II method, estimates component weights that are summed to calculate the aircraft empty weight. Regression equations are used to estimate the weight of each component including structures, propulsion, and systems and equipment. The Roskam method is different from the Raymer and FLOPS methods in that it emphasizes that this level of preliminary analysis is typically inaccurate and therefore recommends averaging all applicable weight estimating relationships that are available to the designer for each component. For simplicity, only the methods presented by Roskam are utilized in this comparison as if this was the only resource available to the designer. For a Boeing 737-200 class aircraft, this typically includes the General Dynamics (GD) method and the Torenbeek method.

While the Roskam approach of averaging multiple methods likely improves the accuracy of this method and makes it easier to tailor the method for different applications, it has the added downside of making the process more cumbersome as each method can have different input parameters, units, and certain methods may group components differently. Additionally, one area of concern with the Roskam method is that if the designer performs a trade study using this method, they must be careful to ensure they have a deep understanding of the input parameters for each method. For example, if a designer is attempting to understand the impact of increasing the distance between the wing and tail quarter mean aerodynamic chord (MAC) on the horizontal tail weight, only the GD method is dependent on that variable. Therefore, any changes to the output of the GD equation will be averaged against the unchanging Torenbeek value, reducing the overall impact. However, one of the advantages of this approach is that it provides a built in error check to the designer. If the result of one method is significantly different from the other, then that is an indication to the designer that there may be a mistake in one of their equations.

\section{Weight Estimation Groups of Interest}

This section includes descriptions of a selection of the weight estimation equations to highlight differences in the methods and the unique features of each. These include the wing, fuselage, vertical tail, horizontal tail, landing gear, fuel system, and avionics system weight estimation methods. While the FLOPS, Raymer, and Roskam methods include provisions to accommodate for different types of aircraft, this discussion remains focused on commercial transport aircraft similar to the Boeing 737-200. Additionally, as they are similar in complexity this comparison is focused on only the FLOPS method, the Raymer third method, and the Roskam Class II method.

All of the methods compared in this section are based on weight estimating regression equations that are a function of parameters that describe the aircraft and its subsystems. Generally, the equations are in exponential form with parameters raised to a power and multiplied, though there are cases where parameters are added or subtracted. Equation 1 is the FLOPS equation for predicting main landing gear weight and is a representative example of the equations used in these methods. 


$$
W L G M=(0.0117-0.0012 * D F T E) * W L D G^{0.95} * X M L G^{0.43}
$$

In this method the weight of the main landing gear is represented by the variable $W L G M$. WLGM is calculated in pounds from the variables DFTE, WLDG, and XMLG. The variable DFTE is used to represent the aircraft type. It is set equal to 1.0 for fighter/attack aircraft and 0.0 for all other aircraft. The variable $W L D G$ is the aircraft landing weight in pounds. The variable $X M L G$ is the length of the main gear oleo strut in inches. Often weight estimating methods will employ significantly more input variables, however the overall approach remains the same. The FLOPS main landing gear weight estimation method will be described in further detail in the following sections of this work.

In the conceptual design process there are unknown aircraft details and the aircraft frequently changes. Therefore, each parameter required by these methods potentially corresponds to additional effort on the part of the designer. The weight estimation methods compared generally require different variables to estimate the same weight items. Therefore, as a way of gauging the level of effort required for each method the parameters required for each method are discussed in this section.

Sensitivity studies are used in this section to highlight the effect of certain variables. These sensitivity studies are conducted by setting up the method of interest for the Boeing 737-200 and changing one parameter to understand how the method responds to those changes. These comparisons are done to highlight the differences in trends between the methods. As there are differences in component groupings and input parameters the results for each method will differ. Therefore, despite the fact that results from all three methods are presented together on a single figure, the reader is discouraged from concluding that one method performs better than the others.

\section{A. Wing Group}

The weight of the wing structure typically forms a large percentage of the aircraft's structural weight, making it critical for the designer to have an accurate estimate of the wing structural weight. However, at this stage in the design cycle the designer could be evaluating thousands or more unique configurations, making it impractical to apply higher order methods such as finite element analysis to accurately estimate the wing structural weight. Therefore, an early weight estimate is crucial to understanding whether or not a concept is feasible. The three methods examined each provide a different approach to estimating wing weight.

The FLOPS wing estimation method was developed to be sensitive to local changes in wing geometry to enable it to be applied to unconventional aircraft. Therefore, the wing weight estimation is the most complex weight estimation method in FLOPS and is the most complex of the three methods compared. The wing weight includes four different sources: the bending material weight; shear material and control surface weight; miscellaneous items weight; and aft body weight for hybrid wing body aircraft. While it would be simpler to combine these into one large equation, this separation provides the designer with more control and insight into the result. For example, if the designer wants to evaluate a technology that will reduce the control surface weight the value can be easily modified to estimate the impact.

The FLOPS wing method has variables and options to allow it to estimate the weight of convention wings, aircraft with strut braced wings, composite wings, wings with aeroelastic tailoring, variable sweep wings, distributed propulsion, wings on aircraft with multiple fuselages, wings that only carry a fraction of the aircraft primary load, and wings on HWB aircraft. Additionally, two different wing definitions are available: detailed and simple. The simple method is comparable to a traditional trapezoidal wing, while the detailed method allows the user to define the wing in more detail by defining details like the engine location, breaks in wing chord, the lift distribution, and the thicknessto-chord ratio distribution. As it is comparable to the other methods the simple method is discussed in this section. Refer to Ref. [3] for a more thorough description of the complex method.

The FLOPS wing weight method is more involved than the other methods discussed here because it involves a few steps. However, each step is not overly complex and could theoretically be simplified down to a single regression equation. The process is as follows:

1) Calculate the bending factor

2) Calculate the bending material weight factor

3) Calculate the shear material and control surface weight

4) Calculate the miscellaneous weight

5) Calculate the bending material weight

6) Calculate the aft body weight (if HWB)

7) Sum the bending material weight, shear material and control surface weight, miscellaneous weight and aft body weight to calculate wing weight

The wing weight is highly dependent on the bending factor that can be estimated with either the simple method or the detailed method. The simple method for estimating the bending factor (Step 1) is a function of the taper ratio, span, 
reference area, whether or not the wing is strut braced, the wing quarter-chord sweep, the aspect ratio, whether or not aeroelastic tailoring has been used in the design of the wing, and the weighted average of the thickness-to-chord ratio.

The bending material factor (Step 2) is calculated from the bending factor, span, ultimate load factor, the degree as to whether composites are used in the wing, whether or not aeroelastic tailoring has been used in the design of the wing, whether or not there are multiple fuselages, whether or not it is a variable sweep wing, and the fraction of the aircraft load carried by the wing. Additionally, if it is a variable sweep wing it is also a function of the wing quarterchord sweep.

The shear material and control surface weight (Step 3) of the wing weight equation is calculated from the degree as to whether composites are used in the wing, the total moveable wing surface area (flaps, elevators, spoilers, etc.), and the design gross weight. The miscellaneous items (Step 4) weight is a function of the degree as to whether composites are used in the wing and the wing reference area.

The reason for the creation of a bending material factor and later calculation of the bending material weight is to account for inertia relief on the bending material weight. Therefore, the bending material weight (step 5) is calculated from the bending material factor, design gross weight, shear material and control surface weight, miscellaneous weight, and the number of wing mounted engines.

As the aft body weight for HWB aircraft term is not distributed to the wings and therefore relevant to the inertia relief process, it is calculated after the bending material weight. It is a function of the number of fuselage mounted engines (scaled to account for distributed propulsion if necessary), the area of the aft body, the design gross weight, the taper ratio of the aft body, and composite utilization factor. Methods are provided in FLOPS to calculate the taper ratio of the aft body if it is not known.

The Raymer wing weight estimation method is more typical of early conceptual design methods. It is based on a single regression equation followed by several adjustment factors that are used to adjust the resulting weight for different options. This equation is dependent on the design gross weight, ultimate load factor, trapezoidal wing area, aspect ratio, the root thickness-to-chord ratio, taper ratio, quarter-chord wing sweep, and wing control surface area. Adjustments for the wing weight are provided to account for the use of advanced composite material, braced wing configuration, and a braced biplane configuration.

The Roskam method for wing weight estimation includes the GD and Torenbeek methods. The GD method provided for the wing group has been excluded from this comparison as it is only valid for aircraft with maximum sea level Mach numbers from 0.4 to 0.8 and is therefore not applicable to most modern commercial airliners. The Torenbeek method includes one equation, the results of which are adjusted based on several design factors. The equation is a function of the maximum zero fuel weight, wing span, semi-chord sweep angle, design ultimate load factor, wing area, and the maximum thickness of the wing root chord. The results of the equation are then adjusted based on the number of wing mounted engines, landing gear location, if the wing is strut braced, and the types of control surfaces used.

Of the three methods presented the FLOPS method is by far the most complicated. However, the additional details provide the designer with more information about the wing, such as how much of the wing weight is due to bending. Despite the added complexity, the method requires roughly only slightly more input parameters than the Raymer and Torenbeek methods.

\section{B. Fuselage Group}

The fuselage structural weight represents a large percentage of the overall aircraft structural weight. Therefore, an accurate estimate of fuselage structural weight is essential early in the conceptual design process. The three methods for fuselage structural weight estimation are described in this section.

The fuselage weight estimation method is typical of FLOPS methods in that it is based on minimal information about the aircraft that is readily available in the preliminary design phase. For commercial aircraft it depends only on fuselage length, average diameter, the number of fuselage mounted engines, the number of fuselages, and whether or not the aircraft is used for military cargo. Additionally, if the average diameter is not known a method is provided to approximate it based on the maximum width and height.

The Raymer fuselage weight estimation method for commercial aircraft requires significantly more input than the FLOPS equation. However, it is unique among the examined methods in that it incorporates parameters from the wing design, which can have a significant effect on fuselage weight. The Raymer method is a regression equation that is a function of whether or not a cargo door is present and its type, if the landing gear is on the fuselage, the design gross weight, the ultimate load factor, the fuselage structural length, the fuselage wetted area, the wing taper ratio, the wing span, the wing quarter-chord sweep, and the fuselage structural depth. Adjustment factors are provided to account for the application of certain materials, if the aircraft is a flying boat, and if the aircraft is carrier based. The material options are advanced composites, wood, and steel, with the default being aluminum. 
The Roskam approach to the fuselage is to average the results from the GD and Torenbeek methods. The GD method is fairly simple in that it only depends on whether the aircraft has engine inlets on the fuselage, the design dive dynamic pressure, the takeoff weight, the length of the fuselage, and the maximum height of the fuselage. The Torenbeek method is dependent on whether or not the fuselage is pressurized, whether or not the main landing gear is mounted to the fuselage, whether or not the aircraft has a cargo floor, the design dive speed, the distance from the wing root quarter-chord to the tail root quarter-chord, the maximum fuselage width, the maximum fuselage depth, and the fuselage gross shell area.

As fuselage depth (or height) can have a relatively complex relationship with fuselage structural weight, fuselage depth proved to be an ideal target for a sensitivity study. To examine how the three methods respond to changes in fuselage depth, they were set up to estimate the weight of the Boeing 737-200 fuselage structure and the fuselage depth was varied. To isolate the effect of a minor change in fuselage depth all other input parameters were held constant, including fuselage wetted area. Therefore, this sensitivity analysis should only be considered valid for slight changes in depth. The results of this sensitivity study are displayed in Fig. 1 below.

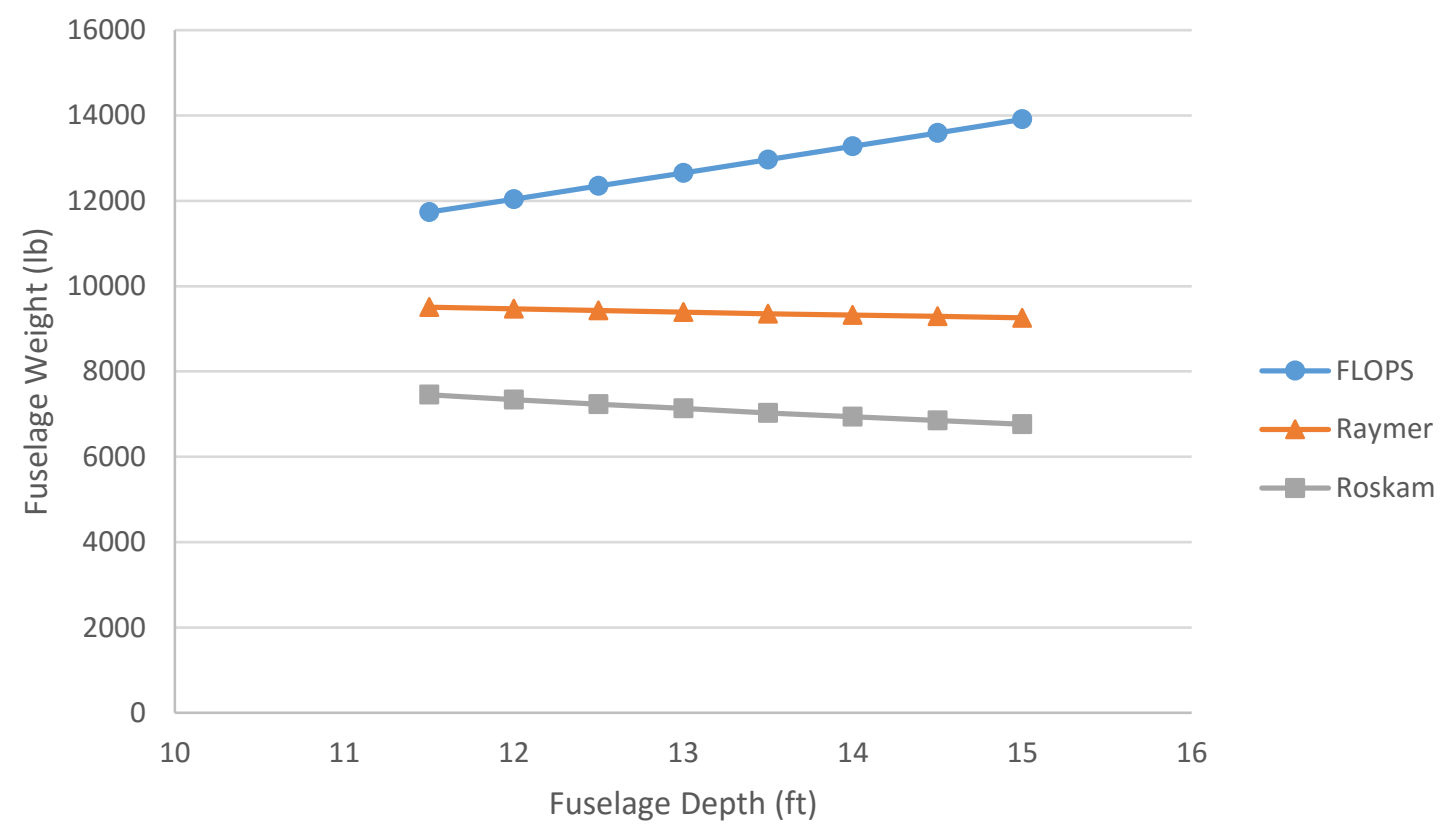

Fig. 1 Sensitivity of fuselage weight to fuselage depth for a Boeing 737-200 style aircraft.

All three methods exhibited a remarkably different response to the change in depth. The FLOPS weight prediction increased with increasing depth, possibly due to the depth only being included through the average diameter parameter. The Raymer and Roskam methods both decreased with increasing fuselage depth. The different responses from the three methods highlight the fact that these methods are not based on the underlying physics behind aircraft design and trade studies should be verified using higher order methods.

\section{Vertical Tail}

The approach used by each method to estimate the vertical tail weight is presented in this section. Estimating the weight of the vertical tail can be especially difficult as the vertical tail weight is highly dependent on the tail geometry and loads. Parameters that represent the tail configuration and tail loading are present in all of the examined methods, however they take on a different form in each.

The FLOPS vertical tail method is a function of the design gross weight, vertical tail theoretical taper ratio, the number of vertical tails, and the vertical tail theoretical area per tail. Additionally, if the vertical tail area is unknown FLOPS provides a novel method for estimating the tail volume coefficient (which can be used to calculate the tail area) based on only aircraft geometry. For additional details on this method refer to Ref. [3]. An interesting feature that sets FLOPS apart from the other two methods is the consideration for aircraft with multiple vertical tails. 
The Raymer vertical tail method includes a regression equation and several adjustment factors. The equation is a function of whether the tail is a T-tail or conventional tail, the flight design gross weight, the ultimate load factor, the distance between the wing quarter-MAC and tail quarter-MAC, the vertical tail area, the aircraft yawing radius of gyration, the vertical tail quarter-chord sweep, the vertical tail aspect ratio, and the root thickness-to-chord ratio. An adjustment factor is provided for the application of advanced composite materials.

For the Roskam method both the GD method and the Torenbeek method were applicable to commercial transport aircraft similar to the Boeing 737-200, meaning that the result is the average of the output from the two methods. The input required for the GD method is the distance from the vertical tail root where the horizontal tail is mounted to the vertical tail, the vertical tail span, the takeoff weight, ultimate load factor, the area of the vertical tail, maximum Mach number at sea level for level flight, distance from the wing quarter mean geometric chord to the vertical tail quarter mean geometric chord, the rudder area, vertical tail aspect ratio, vertical tail taper ratio, and vertical tail quarter-chord sweep. The Torenbeek method depends on whether or not the horizontal tail is mounted to the vertical tail, the vertical tail area, design dive speed, and the vertical tail semi-chord sweep. Additionally, if the horizontal tail is mounted to the vertical tail, then the equation is also dependent on the horizontal tail area, the distance from the vertical tail root where the horizontal tail is mounted to the vertical tail, and the vertical tail span. One of the consequences of incorporating multiple methods is that the GD method requires quarter-chord sweep and the Torenbeek method requires semi-chord sweep. While one value is not hard to calculate from the other, it is a minor difference that can be particularly easy to overlook.

Of the three methods examined, the Raymer method potentially requires significantly more effort than FLOPS or Roskam due to its reliance on the yawing radius of gyration. While Raymer suggests this can be approximated as one third of the distance between the wing quarter-MAC and the tail quarter-MAC, a more accurate determination of this parameter requires the designer to know the mass properties of the aircraft, which can be particularly difficult to estimate early in the conceptual design process.

As all three methods feature the aircraft weight as one of the measures of the load the vertical tail will carry, it is useful to compare how sensitive each method is to changes in design gross weight. Therefore, a basic sensitivity analysis of the three methods was performed by setting up the three methods with values corresponding to the Boeing 737-200 and varying the design gross weight. It is important to note that in order to make this comparison the takeoff gross weight and flight design gross weight were assigned the same values. As the takeoff gross weight refers the to the maximum aircraft weight at takeoff and the flight design gross weight refers to the maximum weight for the aircraft in flight, the latter can often be less. Therefore, while the trends shown in Fig. 2 are representative of the methods, the numerical predictions may be shifted due to this simplification. Figure 2 displays the results of this sensitivity study. The FLOPS and Roskam methods displayed remarkably similar tends, while the Raymer method showed a much greater sensitivity to design gross weight.

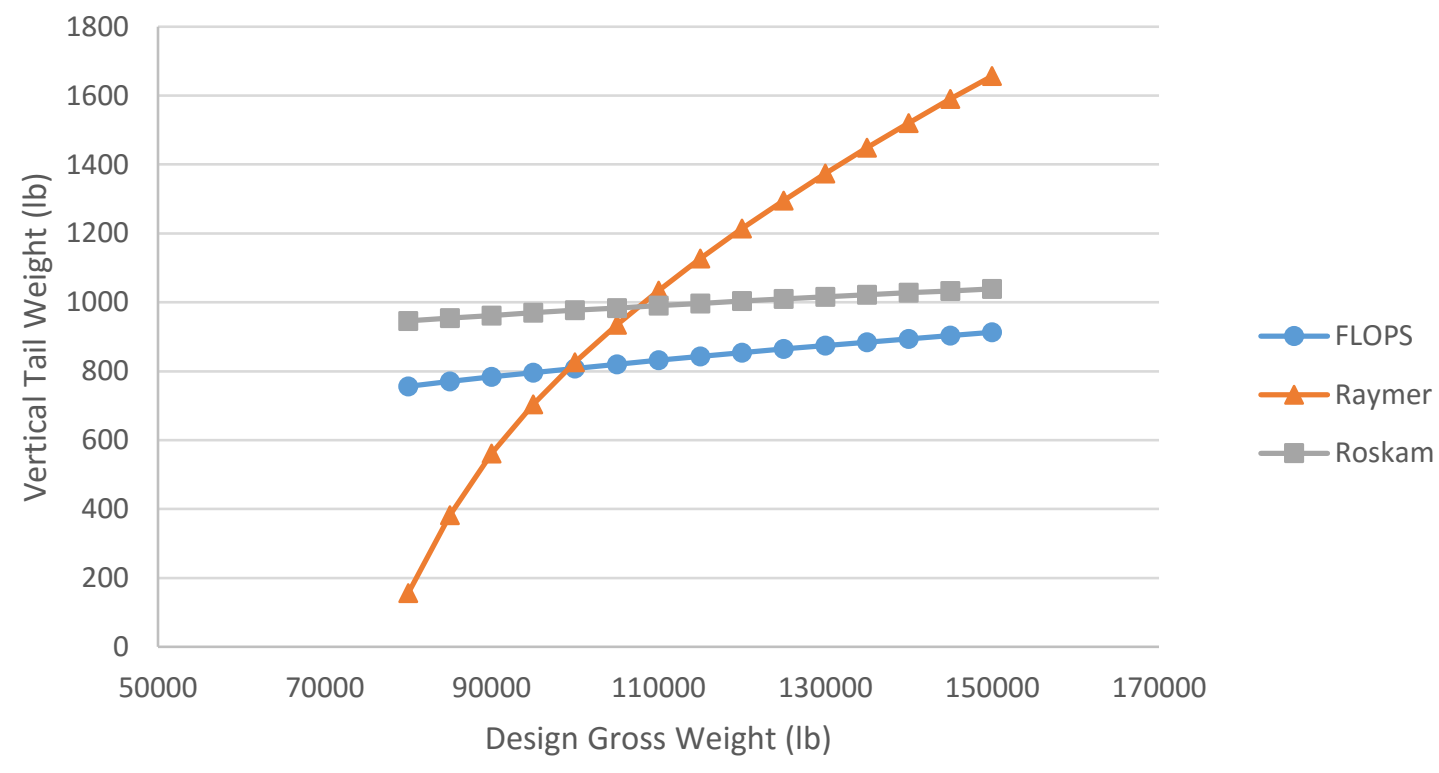

Fig. 2 Sensitivity of vertical tail weight to fuselage depth for a Boeing 737-200 style aircraft. 


\section{Horizontal Tail}

Estimating the weight of the horizontal tail can be especially difficult as the horizontal tail weight is highly dependent on the mass properties of the aircraft and the overall aircraft configuration. This is further complicated by the large number of possible configurations for the horizontal tail such as a conventional tail, T-tail configuration, or an all-moving tail. Parameters that represent the tail configuration and tail loading are present in all of the examined methods. The variables required for each method and its features are described in this section.

The FLOPS method for estimating horizontal tail weight is based on only three variables: the horizontal tail theoretical area, the aircraft design gross weight, and the horizontal tail taper ratio. Additionally, if the horizontal tail area is unknown FLOPS provides a novel method for estimating the tail volume coefficient (which can be used to calculate the tail area) based on only aircraft geometry. For additional details on this method refer to Ref. [3].

Similar to the Raymer method for estimating the vertical tail weight, the Raymer horizontal tail method consists of a regression equation followed by adjustment factors. The regression equation is a function of whether or not the tail is all-moving, the horizontal tail span, the fuselage width at the horizontal tail intersection, the design gross weight, the ultimate load factor, the horizontal tail area, the distance between the wing quarter-MAC and the tail quarterMAC, the aircraft pitching radius of gyration, the horizontal tail quarter-chord sweep, the horizontal tail aspect ratio, and the elevator area. While the pitching radius of gyration can be difficult to calculate for an aircraft concept, a method is provided to estimate it. Adjustment factors are provided for the application of advanced composite materials to the horizontal tail.

For the Roskam method both the GD method and the Torenbeek method were applicable to commercial transport aircraft similar to the Boeing 737-200, meaning that the two methods are used and the result is averaged. The GD and Torenbeek methods each consist of a single equation. The input required for the GD method is the takeoff weight, ultimate load factor, horizontal tail area, horizontal tail span, maximum root thickness of the horizontal tail, mean geometric chord of the horizontal tail, and the distance from the wing quarter mean geometric chord to the horizontal tail quarter mean geometric chord. The inputs required for the Torenbeek method are whether or not the tail has a fixed incidence, the horizontal tail area, the design dive speed, and the horizontal tail semi-chord sweep angle.

As with the previous comparison of the vertical tail methods, the Raymer method potentially requires significantly more effort than the FLOPS or Roskam methods due to its reliance on the pitching radius of gyration. While Raymer suggests this can be approximated as one third of the distance between the wing quarter-MAC and the tail quarterMAC, a more accurate determination of this variable requires the designer to know the mass properties of the aircraft, which can be particularly difficult to estimate early in the conceptual design process.

\section{E. Landing Gear}

The landing gear weight is largely dependent on overall vehicle design factors such as center of gravity, aircraft weight, landing speed, in addition to design factors of the landing gear itself. The three approaches to landing gear weight estimation are presented in this section.

The FLOPS methodology for estimating the landing gear weight includes separate equations for the main and nose gears. The main landing gear weight is a function of whether or not the aircraft is a fighter/attack aircraft, the design landing weight, and the length of the extended main gear oleo strut. The nose gear weight is based on whether or not the aircraft is a fighter/attack aircraft, the design landing weight, the length of the extended nose gear oleo strut, and whether or not the aircraft is carrier based. Additionally, if the values are unknown methods are provided to estimate the design landing weight and the lengths of the extended main and nose gear oleos strut.

The Raymer methodology requires significantly more detailed information about the landing gear design and vehicle loads than either the FLOPS or Roskam approach. The Raymer methodology for estimating landing gear weight includes separate equations for the main and nose gears followed by the application of adjustment factors. The main gear is a function of whether or not it is a kneeling gear, the landing design gross weight, the ultimate landing load factor, the extended length of the main landing gear, the number of main wheels, the number of main gear shock struts, and the aircraft stall speed. The nose gear is a function of whether or not it is a kneeling gear, the landing design gross weight, the ultimate landing load factor, the extended length of the nose gear, and the number of nose wheels. Adjustment factors are available for carrier-based aircraft, and the application of advanced composite materials to the landing gear.

Unlike the FLOPS and Raymer methods the Roskam method calculates the combined weight of the main and nose gears instead of calculating separate weights for each. This requires the designer to estimate a split between the main and nose gears before being able to estimate overall aircraft mass properties. The Roskam method consists of the GD method and the Torenbeek method. The GD method consists of one equation that is a function of only the takeoff weight. The Torenbeek method consists of one equation that is a function of whether or not the wing is low or high and the takeoff weight. 
The methods compared here all include variables that correspond to the load that each landing gear will have to carry and the landing gear configuration. To represent the load that the landing gear will carry, the GD and Torenbeek methods use the takeoff weight while FLOPS uses the landing weight. Raymer is unique in that it incorporates not only the landing weight, but also a landing load factor and the aircraft stall speed. The aircraft stall speed is likely included because it is correlated to landing speed.

Examining how the three methods approximate the configuration of the landing gear provides a good example to show how increasing detail results in increased effort on the part of the designer. All three methods include elements of the landing gear configuration with varying levels of detail. The Roskam method is the simplest in that it only requires whether the wing is high or low, presumably because a high wing results in a longer main landing gear. FLOPS is more direct in that it requires the length of each landing gear oleo strut. Raymer takes the idea a bit further and requires the length of the gear, number of tires, and number of struts. One can see that with each increase in requirements the difficulty in adequately applying the method increases.

\section{F. Fuel System}

The three methods for estimating the weight of the fuel system are detailed in this section. The fuel system weight estimation provides an interesting comparison between the three methods because the fuel system is a fairly complex component of the propulsion system and depends on the overall propulsion system configuration.

The FLOPS fuel system weight includes the weight of fuel tanks and necessary plumbing. It is calculated from the aircraft maximum fuel capacity in terms of weight, the number of engines scaled for distributed propulsion if applicable, and the maximum Mach number. One difference between this method and the others presented is the absence of the number of fuel tanks, which could be a distinct advantage early in the conceptual design phase as there can still be significant change in the overall vehicle configuration.

The Raymer fuel system weight estimation method is a function of the total fuel volume, the volume of any integral tanks, the volume of any self-sealing protected tanks, and the overall number of tanks. Integral tanks are fuel tanks with walls that are formed by structural components. One of the differences between this method and the others is its dependence on fuel volume rather than weight. A unique feature of this method is the ability to divide the fuel between self-sealing and integral tanks, which while not directly applicable to most commercial airliners provides the designer with the ability to easily perform a trade study if such a feature might be useful.

The Roskam method includes separate equations for a fuel system with integral tanks, self-sealing bladder cells, and non-self-sealing bladder cells. Additionally, if the fuel system uses bladder cells a method is provided to estimate the weight of the bladder support structure. The weight estimation for a fuel system with integral tanks uses the provided Torenbeek method and is based on the number of engines, number of separate fuel tanks, the mission fuel weight (including reserves), and the specific weight of the fuel used. In the equation the fuel weight and specific weight are only used together to calculate the fuel volume, indicating that the fuel system weight is a function of fuel volume rather than fuel weight. Specific weights were provided for aviation gasoline and JP-4, and no information is provided as to whether or not the equation is applicable to other propellants. One difference between the Roskam method and the other method is the dependence on mission fuel weight instead of total fuel capacity. While this is often similar to total capacity, there can be significant differences between these values.

While all three methods require a parameter indicating the amount of fuel to be carried, all three methods define this parameter differently: FLOPS uses the maximum fuel weight, Raymer uses maximum fuel volume, and Roskam uses mission fuel capacity. In order to better understand the effect that the amount of fuel has on the fuel system weight, a sensitivity study using parameters from the Boeing 737-200 was performed. This comparison was made using the maximum fuel capacity of the aircraft. As the Roskam method relies on mission fuel capacity (which is typically significantly less than maximum fuel capacity) its results were heavier than they would normally be. However, the overall trend for the Roskam method remained the same. As is shown in Fig. 3, all three methods showed a remarkably similar trend between fuel capacity and fuel system weight. 


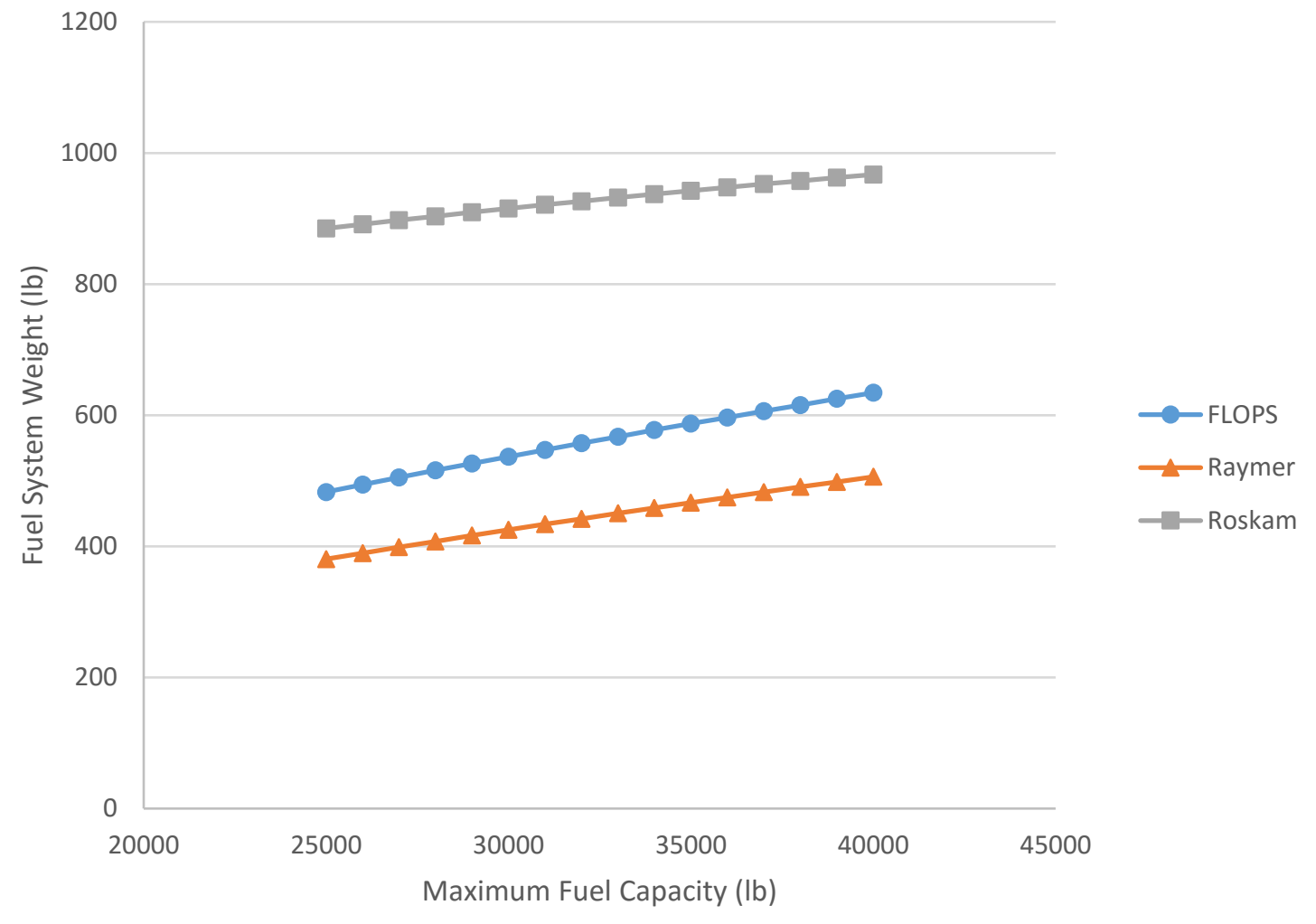

Fig. 3 Sensitivity of fuel system weight to maximum fuel capacity for a Boeing 737-200 style aircraft.

Due to the intended use of FLOPS being the development of advanced aircraft concepts, it has a method built in to account for distributed propulsion. When more than four engines are used on a concept it is assumed that technologies to reduce the penalties associated with the number of engines are also applied. Therefore, the penalties for the number of engines are reduced for certain components. For more details on the distributed propulsion scaling method refer to Ref [3].

As the fuel system is subject to the reduced penalty, the effects of the distributed propulsion scaling are highlighted in the following sensitivity study of the fuel system weight with respect to the number of engines. The results of this study are displayed in Fig. 4. Before the distributed propulsion scaling take effect (four or less engines) both FLOPS and Roskam showed a similar trend. However once the number of engines was increased past four engines the slope of the FLOPS line began to decrease significantly while the slope of the Roskam line showed no change. No change was seen in the Raymer method as it is not a function of the number of engines. 


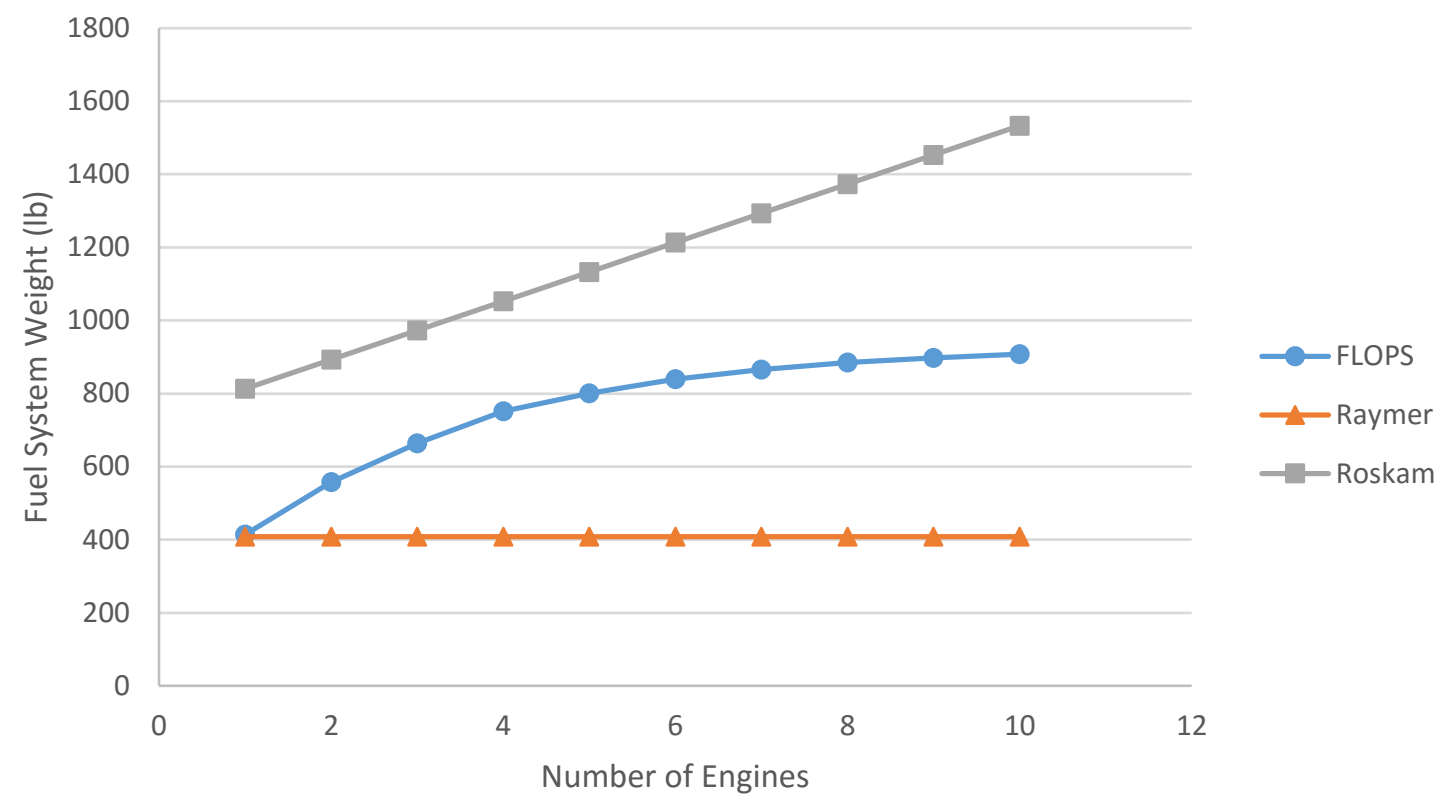

Fig. 4 Sensitivity of fuel system weight to the number of engines for a Boeing 737-200 style aircraft.

\section{G. Avionic System Weight}

The avionics systems weight group highlights one of the principle challenges with comparing these methods, that often the groupings of systems differ from method to method. This not only makes direct comparisons between the methods often meaningless, but also increases the opportunity for error. In this case the Roskam method groups the avionics system with the instrumentation, while the FLOPS and Raymer methods provide separate equations for avionics and instrumentation.

The FLOPS method to estimate avionics system weight is a function of the design range of the aircraft, the number of flight crew, and the fuselage planform area. Additionally, methods are provided to estimate the number of flight crew and fuselage planform area if they are unknown.

The Raymer method for estimating avionics system weight is divided into two steps. First, the uninstalled avionics system weight is estimated. Raymer encourages the designer to use the weight of the specific system that will be used on the aircraft, but as that is not always practical Raymer suggests a range of values for the uninstalled avionics weight. Additionally, methods for estimating this weight are provided in Raymer's second method. The installed avionics weight is then calculated as a function of only the uninstalled avionics weight.

The Roskam method for calculating avionics system weight also includes instrumentation and electronics (not to be confused with the electrical system weight which is calculated separately). No GD method was provided, therefore only the Torenbeek method was used. The Torenbeek method for estimating the weight of the instrumentation, avionics, and electronics is a function of the empty weight and the aircraft maximum range.

\section{FLOPS Weight Predictions}

This section includes comparisons of predictions made with the FLOPS weight methodology with weights from existing aircraft. This section begins with a complete weight buildup of the Boeing 737-200 using the FLOPS detailed wing method. Later in the section FLOPS weight predictions for structural components and subsystems with are compared with results from several existing aircraft.

\section{A. Boeing 737-200 Weight Buildup}

A weight buildup of the Boeing 737-200 was performed using the FLOPS weight estimation method using the detailed wing definition. The results from FLOPS are compared to actual weights in Table 1. For this estimation actual engine weight, payload weight, and gross weight were specified. The Boeing 737-200 structural, propulsion, and systems and equipment weights are from Ref. [5]. The operating empty weight, payload, mission fuel, and gross weight are from Ref. [7]. 
Table 1 Boeing 737-200 FLOPS weight buildup summary.

\begin{tabular}{|c|c|c|c|c|}
\hline & \multicolumn{4}{|c|}{ Boeing 737-200 } \\
\hline & FLOPS (lb) & Actual (lb) & Delta (lb) & Error $(\%)$ \\
\hline Wing & 10,165 & 10,613 & -448 & $-4.2 \%$ \\
\hline Empennage & 2,382 & 2,718 & -336 & $-12.4 \%$ \\
\hline Fuselage & 12,246 & 12,108 & 138 & $1.1 \%$ \\
\hline Landing Gear & 4,497 & 4,354 & 143 & $3.3 \%$ \\
\hline Nacelle & 1,039 & 1,392 & -353 & $-25.4 \%$ \\
\hline Structure Total & 30,329 & 31,185 & -856 & $-2.7 \%$ \\
\hline Engines & 6,217 & 6,217 & 0 & $0.0 \%$ \\
\hline Thrust Reversers & 986 & 1,007 & -21 & $-2.1 \%$ \\
\hline Miscellaneous Systems & 219 & 378 & -159 & $-42.1 \%$ \\
\hline Fuel System-Tanks and Plumbing & 553 & 575 & -22 & $-3.8 \%$ \\
\hline Propulsion Total & 7,975 & 8,177 & -202 & $-2.5 \%$ \\
\hline Surface Controls & 1,291 & 2,348 & $-1,057$ & $-45.0 \%$ \\
\hline Auxiliary Power & 802 & 836 & -34 & $-4.1 \%$ \\
\hline Instruments & 420 & 956 & -536 & $-56.1 \%$ \\
\hline Hydraulics & 826 & 873 & -47 & $-5.4 \%$ \\
\hline Electrical & 1,633 & 1,066 & 567 & $53.2 \%$ \\
\hline Avionics & 1,144 & 625 & 519 & $83.0 \%$ \\
\hline Furnishings and Equipment & 9,328 & 6,643 & 2,685 & $40.4 \%$ \\
\hline Air Conditioning + Anti-Icing & 1,445 & 1,416 & 29 & $2.0 \%$ \\
\hline Miscellaneous & & 124 & & \\
\hline Systems and Equipment Total & 16,889 & 14,887 & 2,002 & $13.4 \%$ \\
\hline Weight Empty & 55,193 & 54,249 & 944 & $1.7 \%$ \\
\hline Crew and Baggage & 915 & & & \\
\hline Unusable Fuel & 342 & & & \\
\hline Engine Oil & 83 & & & \\
\hline Passenger Service & 1,471 & & & \\
\hline \multicolumn{5}{|l|}{ Cargo Containers } \\
\hline Operating Items Total & 2,811 & & & \\
\hline Operating Weight & 58,004 & 60,170 & $-2,166$ & $-3.6 \%$ \\
\hline Passengers & 19,154 & & & \\
\hline Passenger Baggage & 4,532 & & & \\
\hline \multicolumn{5}{|l|}{ Cargo } \\
\hline Payload Items Total & 23,686 & 23,686 & $\mathbf{0}$ & $\mathbf{0 . 0 \%}$ \\
\hline Zero Fuel Weight & 81,690 & 83,856 & $-2,166$ & $-2.6 \%$ \\
\hline Mission Fuel & 33,812 & 31,644 & 2,168 & $6.9 \%$ \\
\hline Ramp (Gross) Weight & 115,502 & 115,500 & 2 & $0.0 \%$ \\
\hline
\end{tabular}


The results show the FLOPS weight estimation method prediction is within four percent of the actual weight for zero fuel, operating, and empty weight. This close agreement is largely expected because data from the Boeing 737 200 or very similar aircraft was likely used in the calibration of the FLOPS weight equations. The estimate is close in other groups, but it is clear that the aircraft component weights are not completely accurate. For example, the FLOPS electrical system weight estimate is 53\% larger than the actual system weight, whereas the instruments weight estimate is $56 \%$ smaller. However, it is possible that some of these errors are due to differences in component groupings.

\section{B. Component Weight Comparisons}

In this section the FLOPS methods were applied to a set of existing aircraft and the result was compared with the actual value. The aircraft included in the comparison included a selection of commercial transport and military aircraft developed between the 1940s and 1970s. The exact aircraft used in the comparison changes from section to section due to missing data on certain aircraft.

1. Wing Group

A wing weight estimation using the simplified wing definition was performed using the parameters from 22 aircraft. The results of this estimation are provided in Fig. 5. Figure 5 is a plot of actual wing weight against FLOPS predicted wing weight. Each point represents an aircraft and the dashed line represents the ideal case of an exact match between predicted and actual, or ' $y=x$ '. A point above or below the line indicates an over prediction and under prediction respectively. The wing weight estimation method manages to capture the overall trends in aircraft weight fairly well with an average error of only $14.2 \%$. There are a few outliers as indicated by points far away from the line, however that is not unexpected for a conceptual design tool.

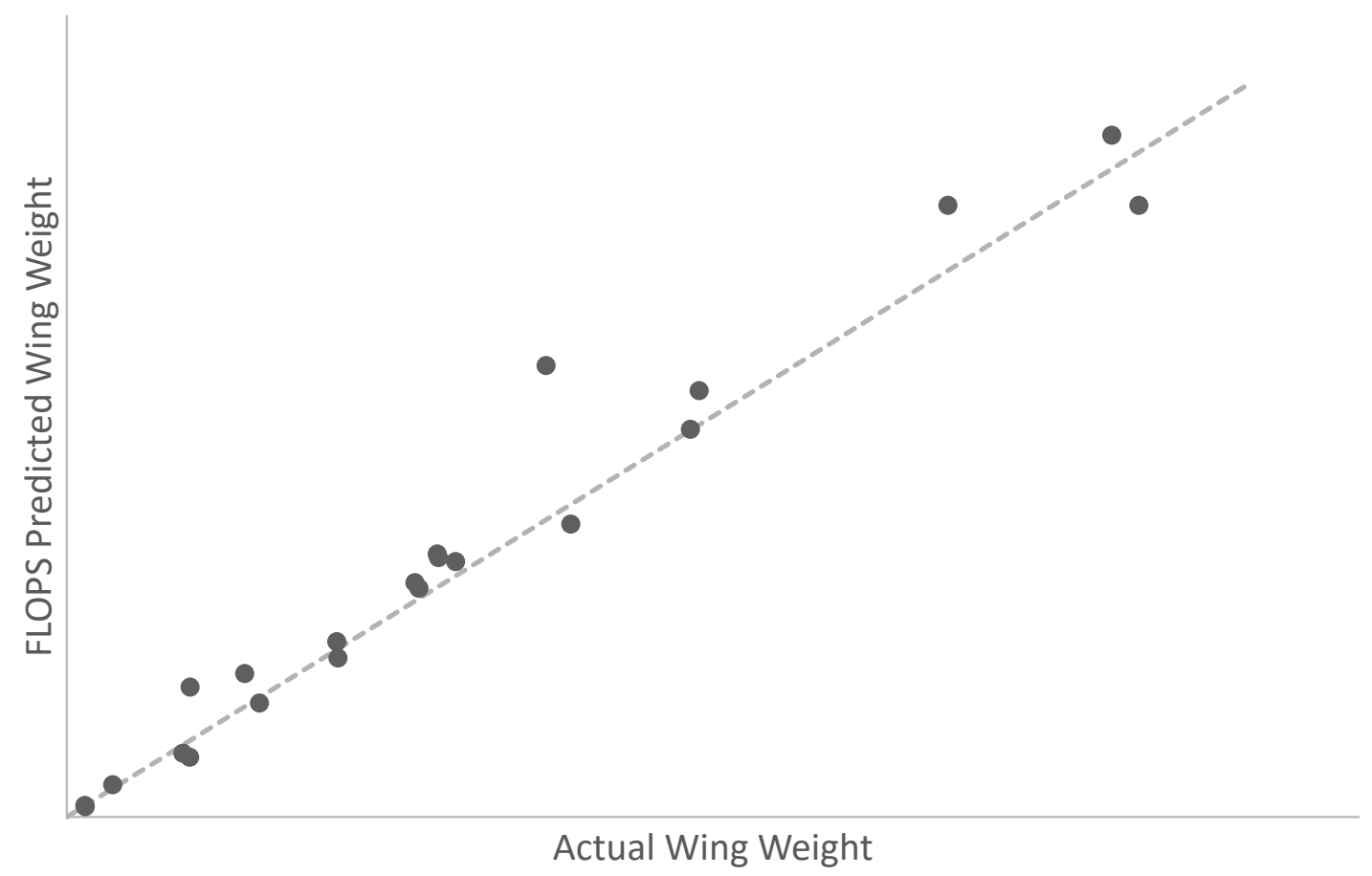

Fig. 5 Comparison of FLOPS calculated wing weight using the simplified wing definition and the actual aircraft wing weight.

\section{Fuselage Group}

A weight estimation using the FLOPS fuselage weight estimation method was performed using the parameters from 20 transport aircraft. The results of this fuselage weight estimation method are plotted in Fig. 6 along with the actual weights for these aircraft. Despite the limited number of input parameters required by the method, it performed well and managed to capture the overall trend in fuselage weight with an average error of only $15.9 \%$. 


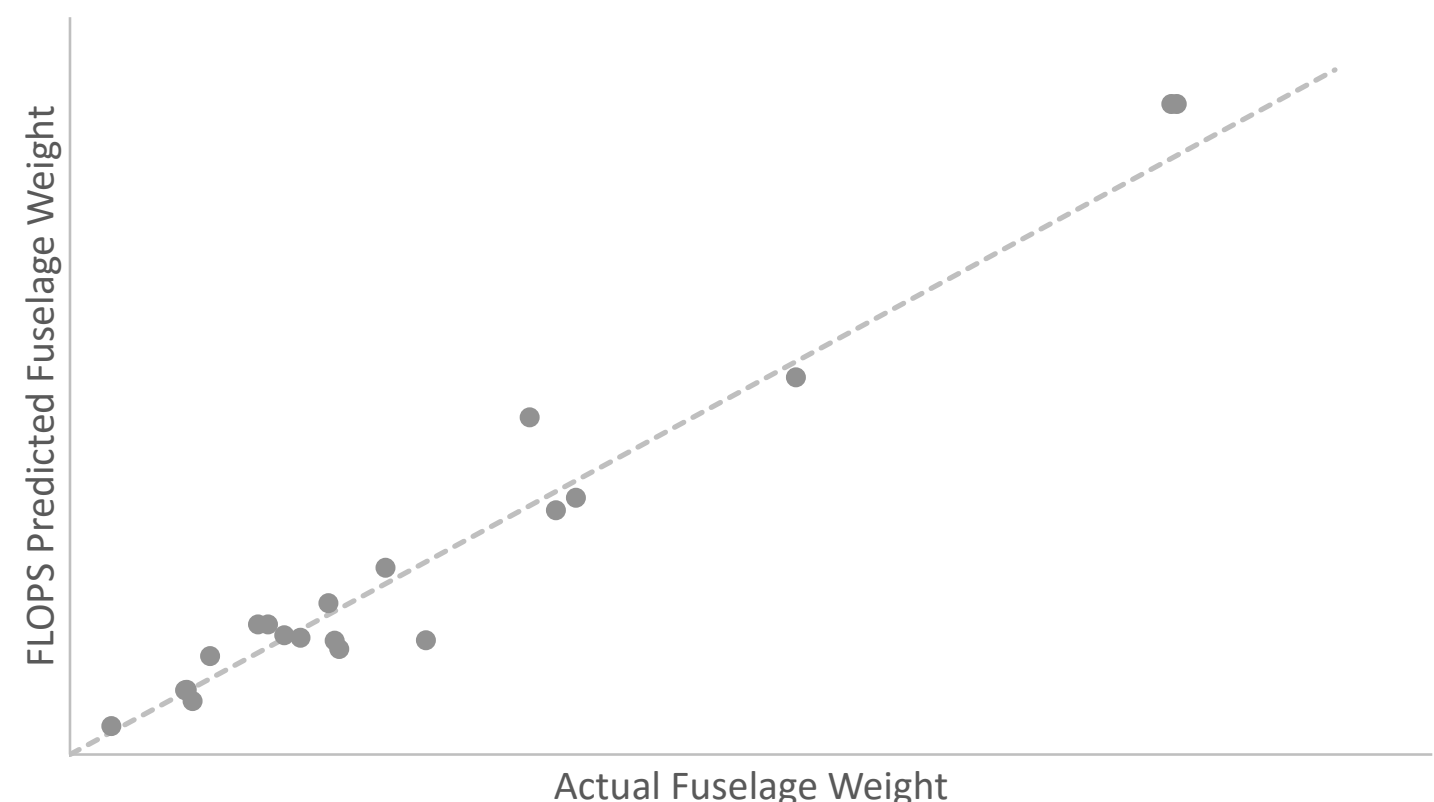

Fig. 6 Comparison of FLOPS predicted fuselage weight versus actual aircraft fuselage weight.

3. Vertical Tail

A weight estimation using the FLOPS vertical tail weight estimation method was performed using the parameters from 23 transport aircraft. The results are plotted in Fig. 7 along with actual weights for the aircraft. This figure shows that the method does a decent job of capturing the overall trends with the number of points above and below the line roughly equal. However, there were several aircraft that this method provided a poor prediction for, as indicated by the points far away from the line. The average prediction error for the selected aircraft was $21.1 \%$.

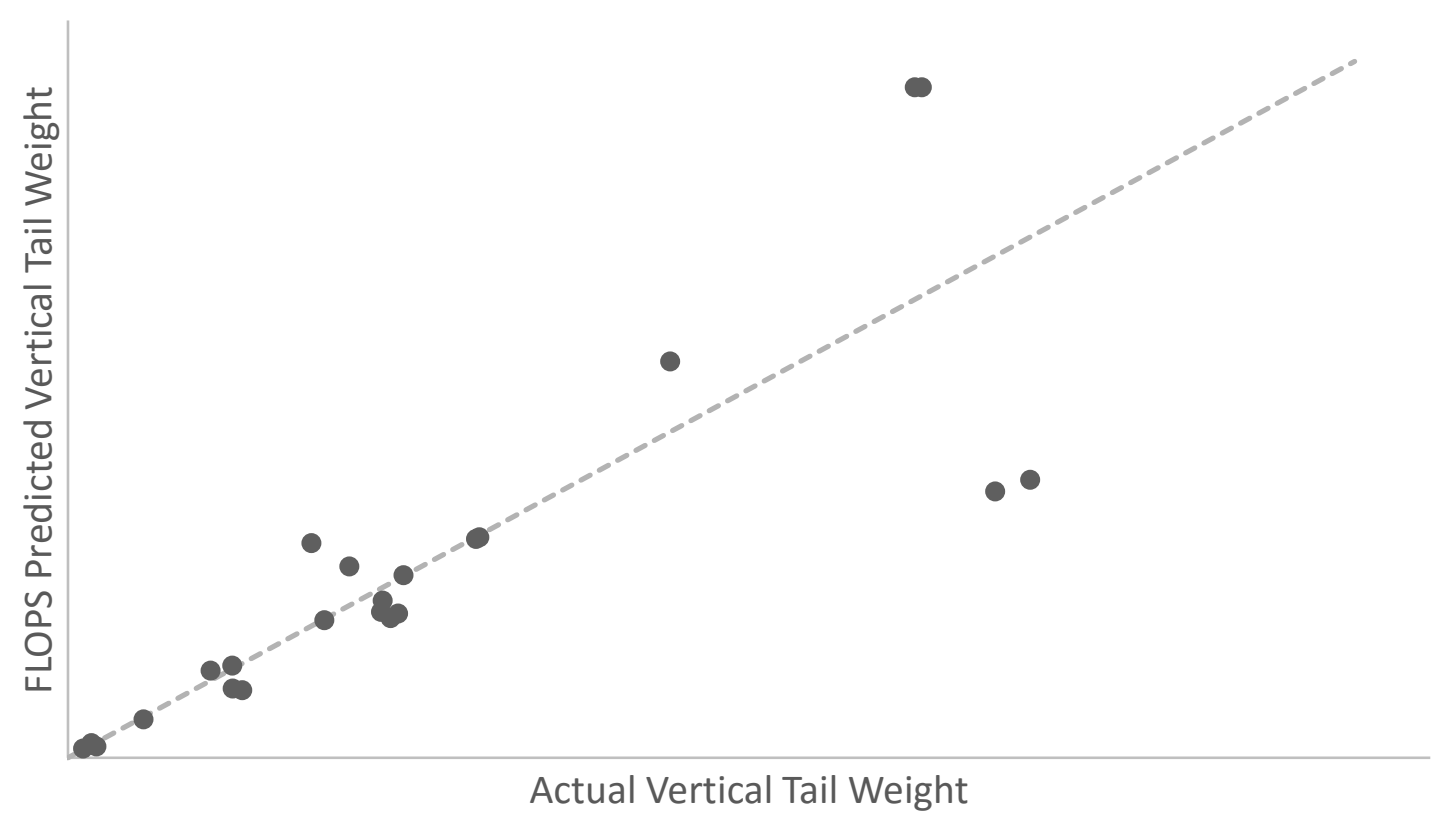

Fig. 7 Comparison of FLOPS predicted vertical tail weight and actual vertical tail weight. 


\section{Horizontal Tail}

A weight estimation using the FLOPS horizontal tail weight estimation method was performed using the parameters from 23 transport aircraft. The results of the FLOPS horizontal tail weight estimation method are plotted in Fig. 8 against the actual weights for these aircraft. This figure shows that the method provided a fairly accurate prediction of horizontal tail weight as indicated by the tight grouping of points around the dashed line. The average error for the selected aircraft was only $10.3 \%$.

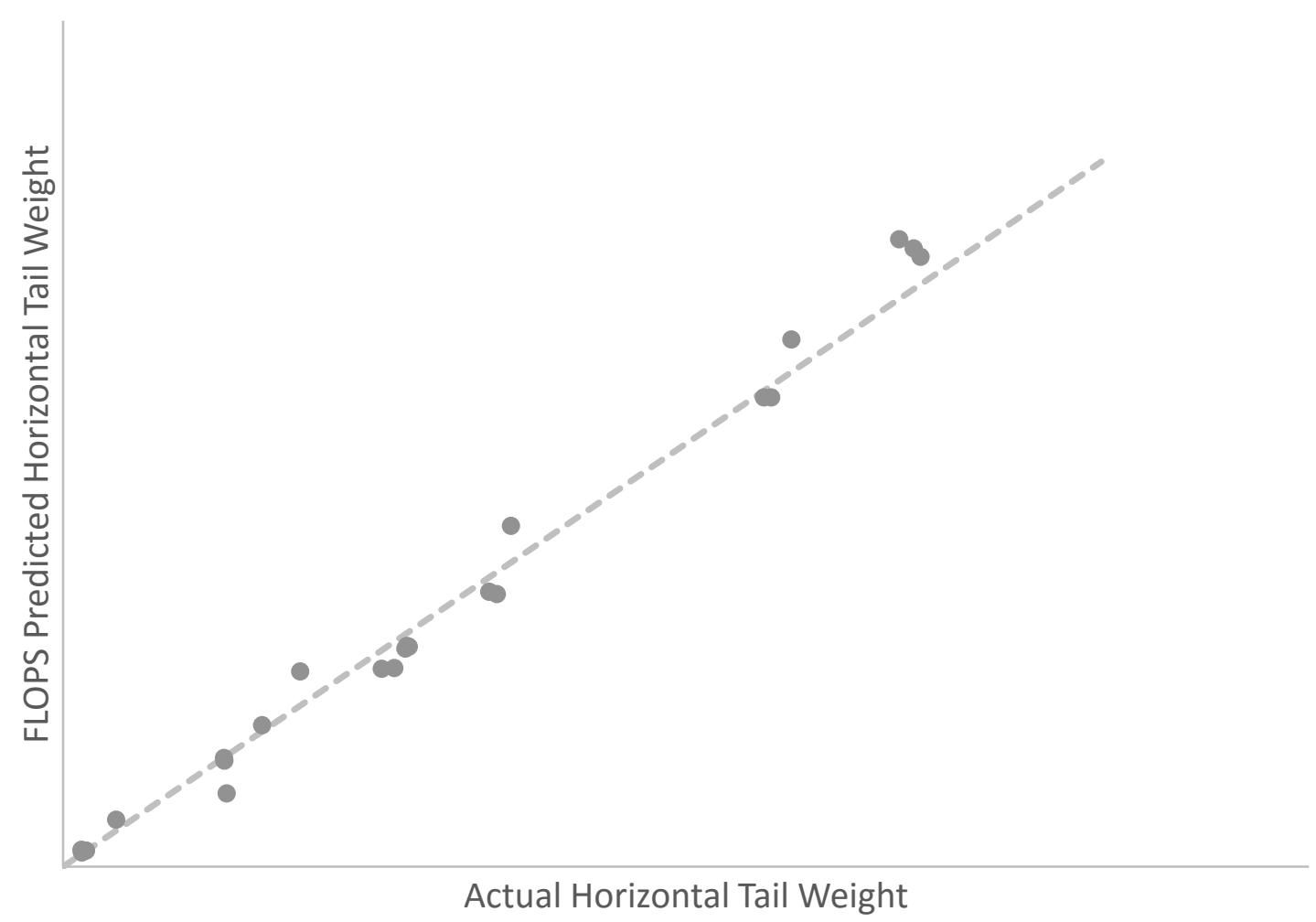

Fig. 8 Comparison of FLOPS predicted horizontal tail weight and actual horizontal tail weight.

\section{Landing Gear}

A weight estimation using the FLOPS main and nose gear weight estimation methods was performed using the parameters from 23 transport aircraft. Results for both the main and nose gear weights are plotted below in Fig. 9 against the actual component weight for each aircraft. For the selected aircraft, the main gear weight estimation method provided a relatively good estimate with an average error of only $11.0 \%$. However, the nose gear method performed poorly when applied larger nose gears, which is reflected in $26.6 \%$ average error. 

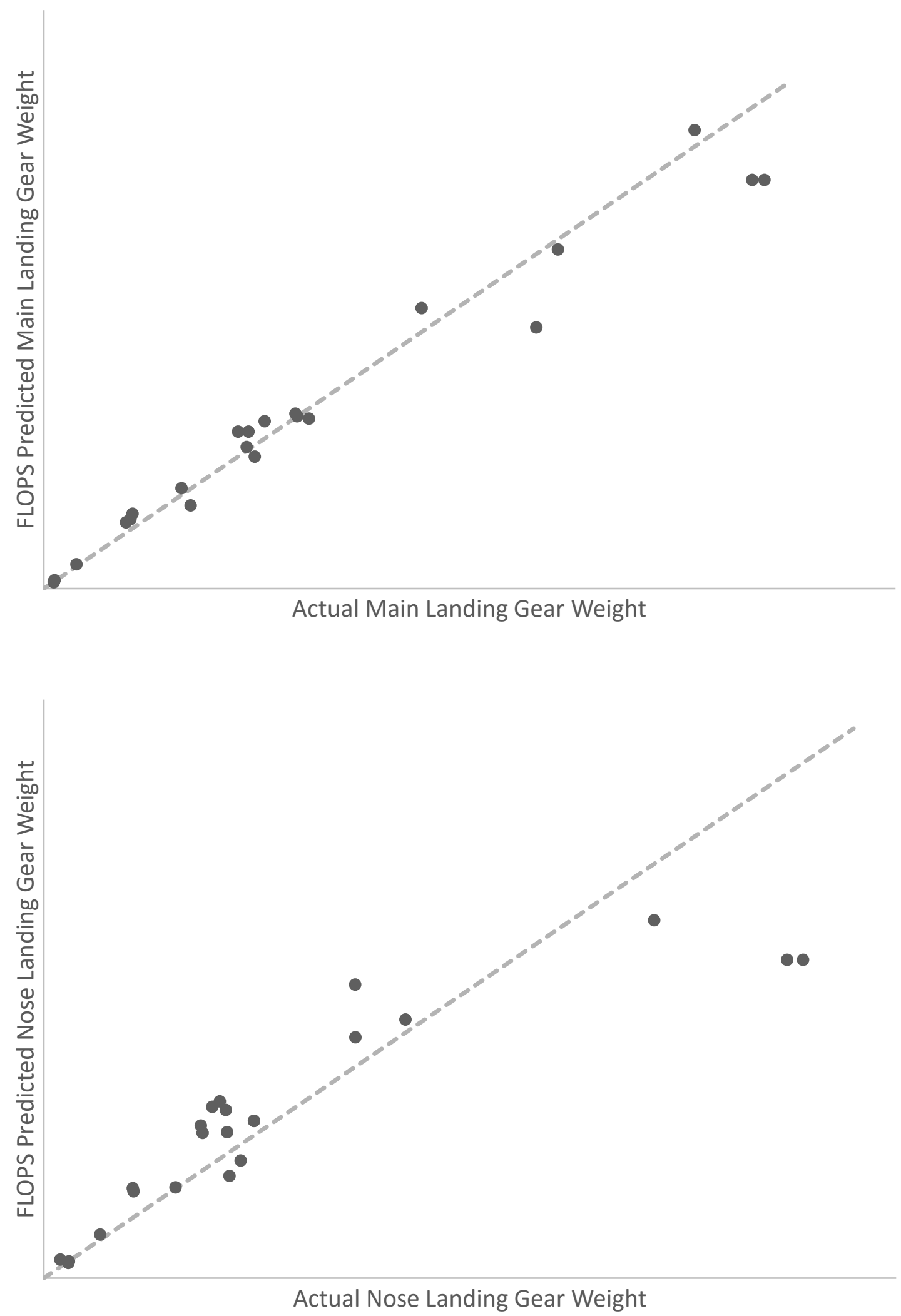

Fig. 9 Comparison of FLOPS predicted main and nose gear weights and actual main and nose gear weights. 


\section{Fuel System}

A weight estimation using the fuel system weight estimation method was performed using the parameters from 21 transport aircraft. Results for FLOPS fuel system weight are shown below in Fig. 10 along with actual component weights. For the selected aircraft, the FLOPS method seems to have a tendency to under predict the fuel system weight (indicated by a point below the dashed line). This is reflected in the average error of 33.4\%. However, part of the discrepancy could be in difficulties estimating the maximum Mach number for the included aircraft and in differences in what components are included in the fuel system weight.

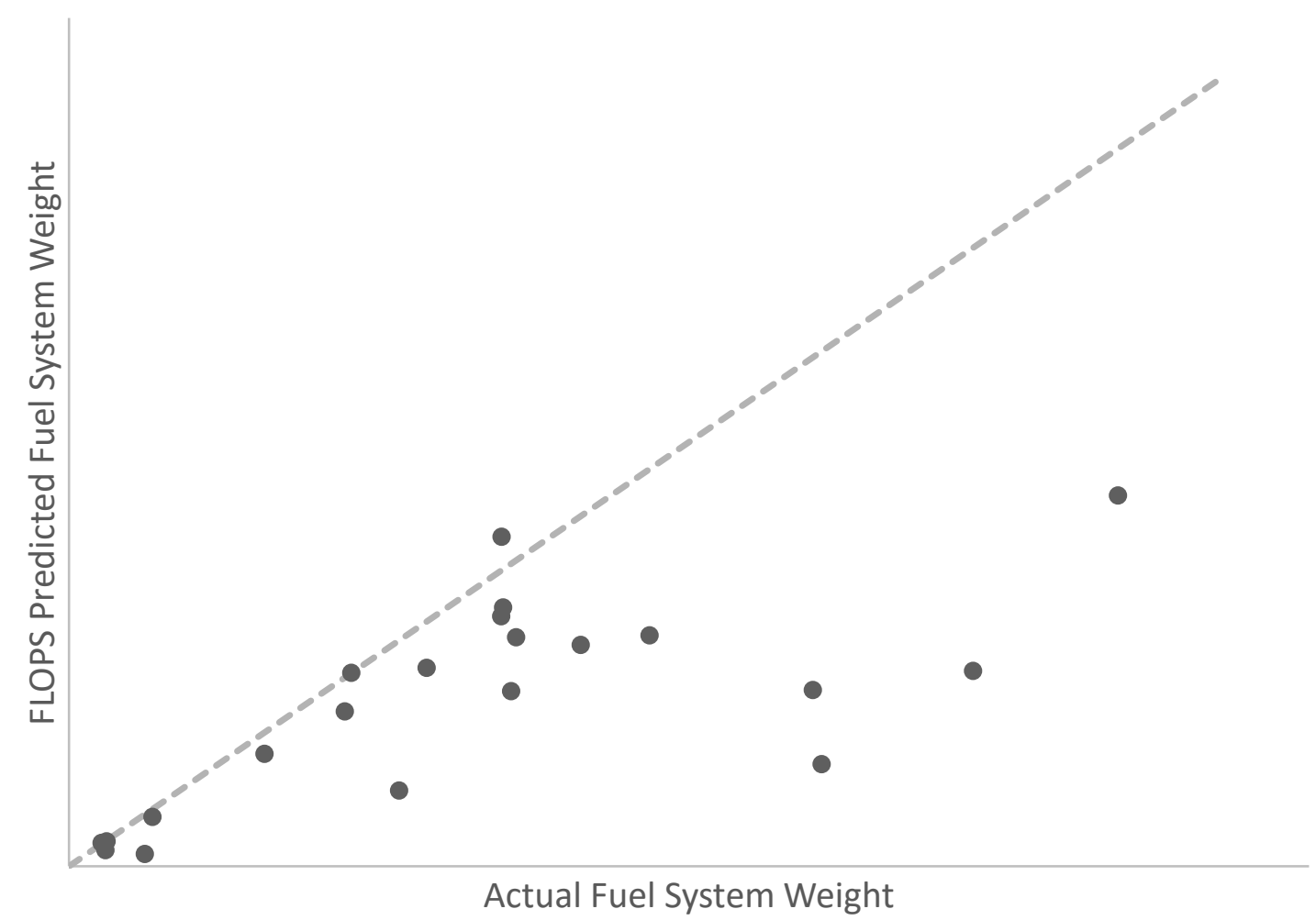

Fig. 10 Comparison between FLOPS predicted and actual fuel system weight.

\section{Avionic System Weight}

An avionics system weight estimation was performed using the parameters from 16 transport aircraft. The results of this estimation are plotted in Fig. 11 against the actual component weight for the aircraft. The method seems to have captured the overall trends in avionics system weight. However, there are a several points that are relatively far away from the line, indicating a poor match between predicted and actual weight. These outlier points lead to an average error of $28.9 \%$. 


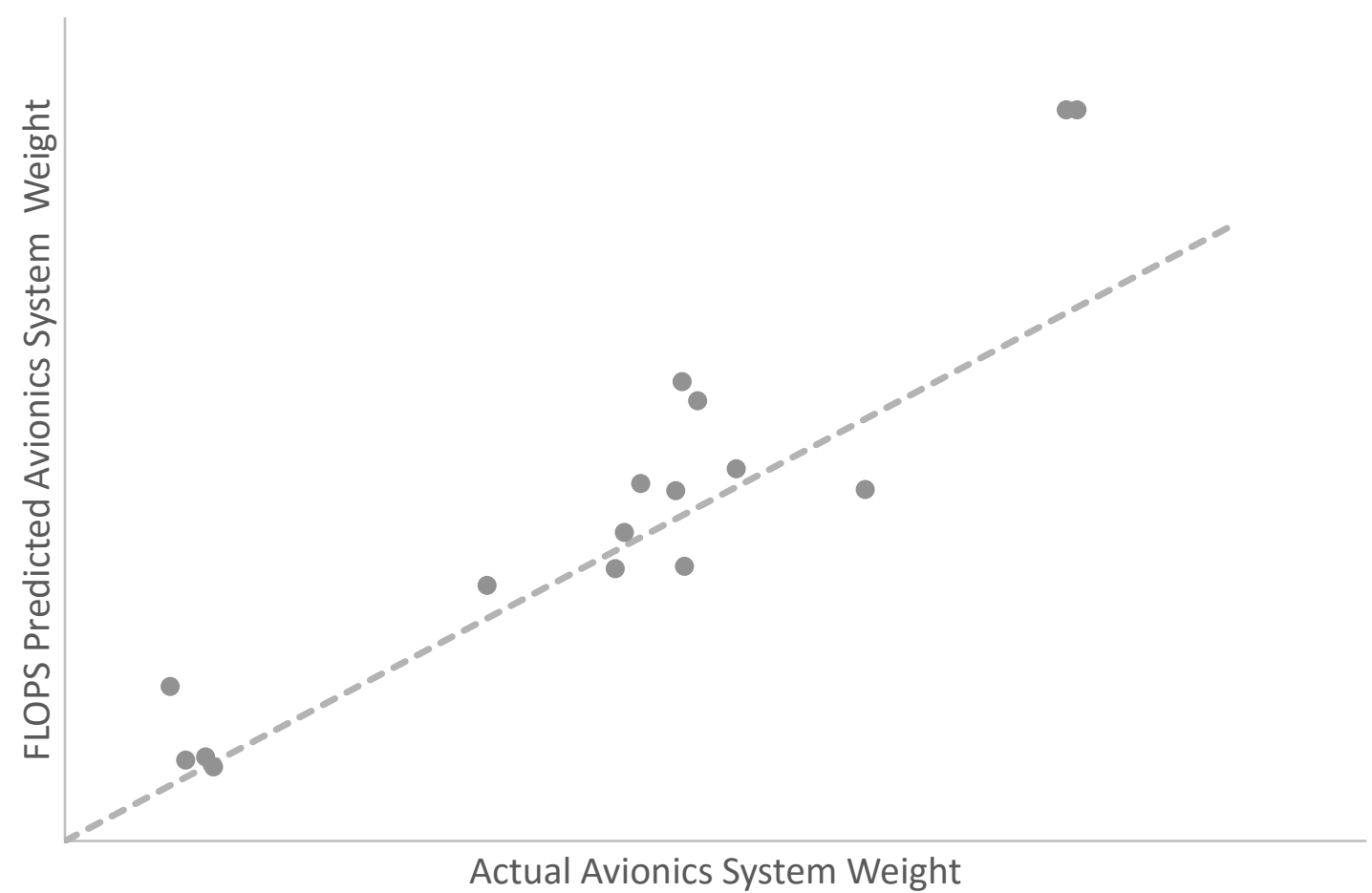

Fig. 11 Comparison of FLOPS predicted avionics system weight and actual avionic system weight.

\section{Conclusions}

The three methods described highlight some of the different approaches in component weight estimation at the conceptual design level. All methods rely on regression equations, however certain methods rely on overall aircraft parameters such as aircraft length, while others rely on parameters that define the component itself such as landing gear length, and some require both.

The FLOPS weight estimation methodology was introduced and shown to be similar to the third Raymer method and the Roskam Class II weight estimation method. Additionally, in most cases the FLOPS method requires a smaller number of input parameters than the other methods and therefore should require less effort on the designer to apply to a concept. Results from applying the FLOPS weight estimation methodology to existing aircraft show that the method adequately captures overall trends in component weight and provides a reasonably accurate prediction of component weight.

Despite all three methods being based on data from actual aircraft, sensitivity studies performed show that they can display remarkably different trends when a single variable is changed. This is a product of how regression equations are typically created to best match a subset of designs with little or no regard to the underlying physics. This indicates that any trade studies performed using these method (or methods of similar type) should be verified with higher order methods. Additionally, the difference in trends lends weight to the Roskam approach of utilizing several of these methods. It significantly increases the work on the designer to apply these tools, but having multiple methods provide different results is a constant reminder that no method should be treated as truth.

A consistent problem encountered while comparing these methods was the lack of thorough documentation. Neither of these methods define the data set that was used in their creation, which has the unfortunate effect of leaving the designer to decide whether or not their concept is applicable (though Roskam does provide some input ranges for select methods). Furthermore, most methods do not provide a thorough description of what is included in each category. For example, the only way a designer would know that the Raymer or FLOPS avionics weight estimation methods do not include instrumentation is because a separate method is provided for instrumentation. There is no documentation spelling this out clearly. This not only leaves the designer to assume what each category includes, but also makes direct comparisons between the methods difficult. While finding non-proprietary information on aircraft 
is often difficult, future methods would be significantly more powerful if they were accompanied by thorough documentation.

\section{Acknowledgments}

This work was completed with support from the Multidisciplinary Design Analysis and Optimization (MDAO) and Systems Analysis theme under the Transformational Tools and Technologies (TTT) project in the Transformative Aeronautics Concepts Program (TACP). The authors would like to thank Mark Guynn, Patricia Glaab, Jason Welstead, and Jeffrey Cerro (NASA Langley Research Center) for their support of this research.

\section{References}

[1] Johnson, V. S., "Minimizing Life Cycle Cost for Subsonic Commercial Aircraft," AIAA Journal of Aircraft, Vol. 27 , No. 2, Feb. 1990, pp. 139-145.

[2] McCullers, L. A., "Aircraft Configuration Optimization Including Optimized Flight Profiles, Multi-disciplinary Analysis and Optimization Part 1", NASA CP-2327, 1984.

[3] Wells, D. P., Horvath, B. L., and McCullers, L. A., “The Flight Optimization System Preliminary Weights Estimation Method," NASA/TM 2017-219627/Volume I, 2017.

[4] Raymer, D. P., Aircraft Design: A Conceptual Approach, $5^{\text {th }}$ ed., AIAA Education Series, AIAA, Reston, VA, 2012.

[5] Roskam, J., Airplane Design: Part V, Component Weight Estimation, Roskam Aviation and Engineering Corporation, Ottawa, KS, 1989.

[6] McCullers, L. A. and Lynch, R. W., "Dynamic Characteristics of Advanced Filamentary Composite Structures, Volume II -Aeroelastic Synthesis Procedure Development", AFFDL-TR-73-111,1974.

[7] 737 Airplane Characteristics for Airport Planning, D6-58325-6, Boeing Commercial Airplanes, Seattle, WA, Sep. 2013. 